DESY-03-059

April 2003

\title{
Measurement of deeply virtual Compton scattering at HERA
}

\author{
ZEUS Collaboration
}

\begin{abstract}
The cross section for deeply virtual Compton scattering in the reaction $e p \rightarrow e \gamma p$ has been measured with the ZEUS detector at HERA using integrated luminosities of $95.0 \mathrm{pb}^{-1}$ of $e^{+} p$ and $16.7 \mathrm{pb}^{-1}$ of $e^{-} p$ collisions. Differential cross sections are presented as a function of the exchanged-photon virtuality, $Q^{2}$, and the centre-of-mass energy, $W$, of the $\gamma^{*} p$ system in the region $5<Q^{2}<100 \mathrm{GeV}^{2}$ and $40<W<140 \mathrm{GeV}$. The measured cross sections rise steeply with increasing $W$. The measurements are compared to QCD-based calculations.
\end{abstract}




\section{The ZEUS Collaboration}

S. Chekanov, M. Derrick, D. Krakauer, J.H. Loizides ${ }^{1}$, S. Magill, B. Musgrave, J. Repond, R. Yoshida

Argonne National Laboratory, Argonne, Illinois 60439-4815 ${ }^{n}$

M.C.K. Mattingly

Andrews University, Berrien Springs, Michigan 49104-0380

P. Antonioli, G. Bari, M. Basile, L. Bellagamba, D. Boscherini, A. Bruni, G. Bruni,

G. Cara Romeo, L. Cifarelli, F. Cindolo, A. Contin, M. Corradi, S. De Pasquale, P. Giusti, G. Iacobucci, A. Margotti, R. Nania, F. Palmonari, A. Pesci, G. Sartorelli, A. Zichichi

University and INFN Bologna, Bologna, Italy ${ }^{e}$

G. Aghuzumtsyan, D. Bartsch, I. Brock, S. Goers, H. Hartmann, E. Hilger, P. Irrgang, H.-P. Jakob, A. Kappes², U.F. Katz ${ }^{2}$, O. Kind, U. Meyer, E. Paul ${ }^{3}$, J. Rautenberg, R. Renner, A. Stifutkin, J. Tandler, K.C. Voss, M. Wang, A. Weber ${ }^{4}$

Physikalisches Institut der Universität Bonn, Bonn, Germany ${ }^{b}$

D.S. Bailey ${ }^{5}$, N.H. Brook ${ }^{5}$, J.E. Cole, B. Foster, G.P. Heath, H.F. Heath, S. Robins, E. Rodrigues ${ }^{6}$, J. Scott, R.J. Tapper, M. Wing

H.H. Wills Physics Laboratory, University of Bristol, Bristol, United Kingdom ${ }^{m}$

M. Capua, A. Mastroberardino, M. Schioppa, G. Susinno

Calabria University, Physics Department and INFN, Cosenza, Italy ${ }^{e}$

J.Y. Kim, Y.K. Kim, J.H. Lee, I.T. Lim, M.Y. Pac ${ }^{7}$

Chonnam National University, Kwangju, Korea ${ }^{g}$

A. Caldwell ${ }^{8}$, M. Helbich, X. Liu, B. Mellado, Y. Ning, S. Paganis, Z. Ren, W.B. Schmidke, F. Sciulli

Nevis Laboratories, Columbia University, Irvington on Hudson, New York 10027o

J. Chwastowski, A. Eskreys, J. Figiel, K. Olkiewicz, P. Stopa, L. Zawiejski

Institute of Nuclear Physics, Cracow, Poland ${ }^{i}$

L. Adamczyk, T. Bołd, I. Grabowska-Bołd, D. Kisielewska, A.M. Kowal, M. Kowal, T. Kowalski, M. Przybycień, L. Suszycki, D. Szuba, J. Szuba ${ }^{9}$

Faculty of Physics and Nuclear Techniques, University of Mining and Metallurgy, Cracow, Poland ${ }^{p}$

A. Kotański ${ }^{10}$, W. Słomiński ${ }^{11}$

Department of Physics, Jagellonian University, Cracow, Poland 
V. Adler, L.A.T. Bauerdick ${ }^{12}$, U. Behrens, I. Bloch, K. Borras, V. Chiochia, D. Dannheim, G. Drews, J. Fourletova, U. Fricke, A. Geiser, P. Göttlicher ${ }^{13}$, O. Gutsche, T. Haas, W. Hain, G.F. Hartner, S. Hillert, B. Kahle, U. Kötz, H. Kowalski ${ }^{14}$, G. Kramberger, H. Labes, D. Lelas, B. Löhr, R. Mankel, I.-A. Melzer-Pellmann, M. Moritz ${ }^{15}$, C.N. Nguyen, D. Notz, M.C. Petrucci ${ }^{16}$, A. Polini, A. Raval, U. Schneekloth, F. Selonke ${ }^{3}$, U. Stoesslein, H. Wessoleck, G. Wolf, C. Youngman, W. Zeuner

Deutsches Elektronen-Synchrotron DESY, Hamburg, Germany

S. Schlenstedt

DESY Zeuthen, Zeuthen, Germany

G. Barbagli, E. Gallo, C. Genta, P. G. Pelfer

University and INFN, Florence, Italy ${ }^{e}$

A. Bamberger, A. Benen, N. Coppola

Fakultät für Physik der Universität Freiburg i.Br., Freiburg i.Br., Germany ${ }^{b}$

M. Bell, P.J. Bussey, A.T. Doyle, C. Glasman, J. Hamilton, S. Hanlon, S.W. Lee, A. Lupi, D.H. Saxon, I.O. Skillicorn

Department of Physics and Astronomy, University of Glasgow, Glasgow, United King$\mathrm{dom}^{\mathrm{m}}$

I. Gialas

Department of Engineering in Management and Finance, Univ. of Aegean, Greece

B. Bodmann, T. Carli, U. Holm, K. Klimek, N. Krumnack, E. Lohrmann, M. Milite, H. Salehi, S. Stonjek ${ }^{17}$, K. Wick, A. Ziegler, Ar. Ziegler

Hamburg University, Institute of Exp. Physics, Hamburg, Germany ${ }^{b}$

C. Collins-Tooth, C. Foudas, R. Gonçalo ${ }^{6}$, K.R. Long, A.D. Tapper

Imperial College London, High Energy Nuclear Physics Group, London, United King$d^{m}{ }^{m}$

P. Cloth, D. Filges

Forschungszentrum Jülich, Institut für Kernphysik, Jülich, Germany

K. Nagano, K. Tokushuku ${ }^{18}$, S. Yamada, Y. Yamazaki

Institute of Particle and Nuclear Studies, KEK, Tsukuba, Japan ${ }^{f}$

A.N. Barakbaev, E.G. Boos, N.S. Pokrovskiy, B.O. Zhautykov

Institute of Physics and Technology of Ministry of Education and Science of Kazakhstan, Almaty, Kazakhstan

H. Lim, D. Son

Kyungpook National University, Taegu, Korea ${ }^{g}$ 
K. Piotrzkowski

Institut de Physique Nucléaire, Université Catholique de Louvain, Louvain-la-Neuve, Belgium

F. Barreiro, O. González, L. Labarga, J. del Peso, E. Tassi, J. Terrón, M. Vázquez

Departamento de Física Teórica, Universidad Autónoma de Madrid, Madrid, Spain ${ }^{l}$

M. Barbi, F. Corriveau, S. Gliga, J. Lainesse, S. Padhi, D.G. Stairs

Department of Physics, McGill University, Montréal, Québec, Canada H3A 2T8 ${ }^{a}$

T. Tsurugai

Meiji Gakuin University, Faculty of General Education, Yokohama, Japan ${ }^{f}$

A. Antonov, P. Danilov, B.A. Dolgoshein, D. Gladkov, V. Sosnovtsev, S. Suchkov Moscow Engineering Physics Institute, Moscow, Russia ${ }^{j}$

R.K. Dementiev, P.F. Ermolov, Yu.A. Golubkov, I.I. Katkov, L.A. Khein, I.A. Korzhavina, V.A. Kuzmin, B.B. Levchenko ${ }^{19}$, O.Yu. Lukina, A.S. Proskuryakov, L.M. Shcheglova, N.N. Vlasov, S.A. Zotkin

Moscow State University, Institute of Nuclear Physics, Moscow, Russia ${ }^{k}$

N. Coppola, S. Grijpink, E. Koffeman, P. Kooijman, E. Maddox, A. Pellegrino, S. Schagen, H. Tiecke, J.J. Velthuis, L. Wiggers, E. de Wolf NIKHEF and University of Amsterdam, Amsterdam, Netherlands ${ }^{h}$

N. Brümmer, B. Bylsma, L.S. Durkin, T.Y. Ling Physics Department, Ohio State University, Columbus, Ohio $43210^{n}$

A.M. Cooper-Sarkar, A. Cottrell, R.C.E. Devenish, J. Ferrando, G. Grzelak, S. Patel, M.R. Sutton, R. Walczak Department of Physics, University of Oxford, Oxford United Kingdom ${ }^{m}$

A. Bertolin, R. Brugnera, R. Carlin, F. Dal Corso, S. Dusini, A. Garfagnini, S. Limentani, A. Longhin, A. Parenti, M. Posocco, L. Stanco, M. Turcato Dipartimento di Fisica dell' Università and INFN, Padova, Italy ${ }^{e}$

E.A. Heaphy, F. Metlica, B.Y. Oh, P.R.B. Saull ${ }^{20}$, W.S. Toothacker ${ }^{21}$, J.J. Whitmore ${ }^{22}$ Department of Physics, Pennsylvania State University, University Park, Pennsylvania $16802^{\circ}$

Y. Iga

Polytechnic University, Sagamihara, Japan ${ }^{f}$

G. D’Agostini, G. Marini, A. Nigro

Dipartimento di Fisica, Università 'La Sapienza' and INFN, Rome, Italy ${ }^{e}$ 
C. Cormack ${ }^{23}$, J.C. Hart, N.A. McCubbin

Rutherford Appleton Laboratory, Chilton, Didcot, Oxon, United Kingdom ${ }^{m}$

C. Heusch

University of California, Santa Cruz, California $95064^{n}$

I.H. Park

Department of Physics, Ewha Womans University, Seoul, Korea

N. Pavel

Fachbereich Physik der Universität-Gesamthochschule Siegen, Germany

H. Abramowicz, A. Gabareen, S. Kananov, A. Kreisel, A. Levy

Raymond and Beverly Sackler Faculty of Exact Sciences, School of Physics, Tel-Aviv

University, Tel-Aviv, Israel ${ }^{d}$

M. Kuze

Department of Physics, Tokyo Institute of Technology, Tokyo, Japan ${ }^{f}$

T. Abe, T. Fusayasu, S. Kagawa, T. Kohno, T. Tawara, T. Yamashita

Department of Physics, University of Tokyo, Tokyo, Japan ${ }^{f}$

R. Hamatsu, T. Hirose ${ }^{3}$, M. Inuzuka, S. Kitamura ${ }^{24}$, K. Matsuzawa, T. Nishimura

Tokyo Metropolitan University, Department of Physics, Tokyo, Japan ${ }^{f}$

M. Arneodo ${ }^{25}$, M.I. Ferrero, V. Monaco, M. Ruspa, R. Sacchi, A. Solano

Università di Torino, Dipartimento di Fisica Sperimentale and INFN, Torino, Italy ${ }^{e}$

T. Koop, G.M. Levman, J.F. Martin, A. Mirea

Department of Physics, University of Toronto, Toronto, Ontario, Canada M5S 1A7\%a

J.M. Butterworth, C. Gwenlan, R. Hall-Wilton, T.W. Jones, M.S. Lightwood, B.J. West

Physics and Astronomy Department, University College London, London, United Kingdom ${ }^{m}$

J. Ciborowski ${ }^{26}$, R. Ciesielski ${ }^{27}$, R.J. Nowak, J.M. Pawlak, J. Sztuk ${ }^{28}$, T. Tymieniecka ${ }^{29}$, A. Ukleja ${ }^{29}$, J. Ukleja, A.F. Żarnecki

Warsaw University, Institute of Experimental Physics, Warsaw, Poland ${ }^{q}$

M. Adamus, P. Plucinski

Institute for Nuclear Studies, Warsaw, Poland ${ }^{q}$

Y. Eisenberg, L.K. Gladilin ${ }^{30}$, D. Hochman, U. Karshon M. Riveline

Department of Particle Physics, Weizmann Institute, Rehovot, Israel ${ }^{c}$

D. Kçira, S. Lammers, L. Li, D.D. Reeder, A.A. Savin, W.H. Smith

Department of Physics, University of Wisconsin, Madison, Wisconsin $53706^{n}$ 
A. Deshpande, S. Dhawan, P.B. Straub

Department of Physics, Yale University, New Haven, Connecticut 06520-8121 ${ }^{n}$

S. Bhadra, C.D. Catterall, S. Fourletov, G. Hartner, S. Menary, M. Soares, J. Standage Department of Physics, York University, Ontario, Canada M3J 1 P3 ${ }^{a}$ 
${ }^{1}$ also affiliated with University College London

${ }^{2}$ on leave of absence at University of Erlangen-Nürnberg, Germany

${ }^{3}$ retired

4 self-employed

5 PPARC Advanced fellow

${ }^{6}$ supported by the Portuguese Foundation for Science and Technology (FCT)

7 now at Dongshin University, Naju, Korea

${ }^{8}$ now at Max-Planck-Institut für Physik, München/Germany

${ }^{9}$ partly supported by the Israel Science Foundation and the Israel Ministry of Science

10 supported by the Polish State Committee for Scientific Research, grant no. 2 P03B 09322

11 member of Dept. of Computer Science

12 now at Fermilab, Batavia/IL, USA

13 now at DESY group FEB

14 on leave of absence at Columbia Univ., Nevis Labs., N.Y./USA

15 now at CERN

16 now at INFN Perugia, Perugia, Italy

17 now at Univ. of Oxford, Oxford/UK

18 also at University of Tokyo

19 partly supported by the Russian Foundation for Basic Research, grant 02-02-81023

20 now at National Research Council, Ottawa/Canada

21 deceased

22 on leave of absence at The National Science Foundation, Arlington, VA/USA

23 now at Univ. of London, Queen Mary College, London, UK

24 present address: Tokyo Metropolitan University of Health Sciences, Tokyo 116-8551, Japan

25 also at Università del Piemonte Orientale, Novara, Italy

26 also at Łódź University, Poland

27 supported by the Polish State Committee for Scientific Research, grant no. 2 P03B 07222

28 Eódź University, Poland

29 supported by German Federal Ministry for Education and Research (BMBF), POL $01 / 043$

30 on leave from MSU, partly supported by University of Wisconsin via the U.S.-Israel BSF 
a supported by the Natural Sciences and Engineering Research Council of Canada (NSERC)

$b$ supported by the German Federal Ministry for Education and Research (BMBF), under contract numbers HZ1GUA 2, HZ1GUB 0, HZ1PDA 5, HZ1VFA 5

$c$ supported by the MINERVA Gesellschaft für Forschung GmbH, the Israel Science Foundation, the U.S.-Israel Binational Science Foundation and the Benozyio Center for High Energy Physics

$d$ supported by the German-Israeli Foundation and the Israel Science Foundation

$e$ supported by the Italian National Institute for Nuclear Physics (INFN)

$f$ supported by the Japanese Ministry of Education, Culture, Sports, Science and Technology (MEXT) and its grants for Scientific Research

$g$ supported by the Korean Ministry of Education and Korea Science and Engineering Foundation

$h$ supported by the Netherlands Foundation for Research on Matter (FOM)

$i$ supported by the Polish State Committee for Scientific Research, grant no. 620/E-77/SPUB-M/DESY/P-03/DZ 247/2000-2002

$j$ partially supported by the German Federal Ministry for Education and Research (BMBF)

$k$ supported by the Fund for Fundamental Research of Russian Ministry for Science and Education and by the German Federal Ministry for Education and Research (BMBF)

$l$ supported by the Spanish Ministry of Education and Science through funds provided by CICYT

$m$ supported by the Particle Physics and Astronomy Research Council, UK

$n$ supported by the US Department of Energy

$o$ supported by the US National Science Foundation

$p$ supported by the Polish State Committee for Scientific Research, grant no. 112/E-356/SPUB-M/DESY/P-03/DZ 301/2000-2002, 2 P03B 13922

$q$ supported by the Polish State Committee for Scientific Research, grant no. 115/E-343/SPUB-M/DESY/P-03/DZ 121/2001-2002, 2 P03B 07022 


\section{Introduction}

This paper reports cross-section measurements for the exclusive production of a real photon in diffractive $e p$ interactions, $e p \rightarrow e \gamma p$, as shown in Fig. 17. This exclusive process, known as deeply virtual Compton scattering (DVCS) 1, 2, 3, 4, is calculable in perturbative QCD (pQCD), when the virtuality, $Q^{2}$, of the exchanged photon is large.

The DVCS reaction can be regarded as the elastic scattering of the virtual photon off the proton via a colourless exchange. The pQCD calculations assume that the exchange involves two partons, having different longitudinal and transverse momenta, in a colourless configuration. These unequal momenta are a consequence of the mass difference between the incoming virtual photon and the outgoing real photon. The DVCS cross section depends, therefore, on the generalised parton distributions (GPD) [5, 4, 6, 7, which carry information about the wave function of the proton. The cross section at sufficiently large $Q^{2}$ is expected to rise steeply with increasing $W$, the centre-of-mass energy of the virtual photon-proton system, due to the fast rise of the parton densities in the proton towards smaller $x$ values, where $x$ is the Bjorken scaling variable.

The initial and final states of the DVCS process are identical to those of the purely electromagnetic Bethe-Heitler (BH) process (Figs. 1b,c). The interference between these two processes in principle provides information about the real and imaginary parts of the QCD scattering amplitude [8, 9, 10]. However, it is expected to be small in the kinematic region studied in this paper [8, 9].

The simplicity of the final state and the absence of complications due to hadronisation mean that the QCD predictions are more reliable than for many other exclusive final states. This reaction is one of the theoretically best-understood exclusive QCD processes in ep collisions. The first measurements of the DVCS process at high $W$ [11, 12] and its beam-spin asymmetry in polarised ep scattering at low $W$ [13, 14] have recently become available.

In the analysis presented here, the dependence of the DVCS cross section on $W$ and $Q^{2}$ is studied in the kinematic range $5<Q^{2}<100 \mathrm{GeV}^{2}$ and $40<W<140 \mathrm{GeV}$. The measurements are integrated over $t$, the square of the four-momentum transfer at the proton vertex. The $e^{+} p$ cross sections are based on a ten-fold increase in statistics over a previous HERA result [12], permitting a study of the $W$ dependence of the cross section as well as a significant extension of the $Q^{2}$ range probed. This paper also reports the first measurement of the $e^{-} p$ cross sections. 


\section{Experimental set-up}

The data were collected by the ZEUS detector at HERA during the 96-00 running periods. In 96-97, HERA collided $27.5 \mathrm{GeV}$ positrons with $820 \mathrm{GeV}$ protons. In 98-00, the proton energy was increased to $920 \mathrm{GeV}$ and both positrons and electrons were collided. The measurements for $e^{+} p\left(e^{-} p\right)$ interactions ${ }^{1}$ are based on an integrated luminosity of $95 \mathrm{pb}^{-1}$ $\left(17 \mathrm{pb}^{-1}\right)$.

A detailed description of the ZEUS detector can be found elsewhere [15. A brief outline of the components that are most relevant for this analysis is given below.

Charged particles are tracked in the central tracking detector (CTD) [16, which operates in a magnetic field of $1.43 \mathrm{~T}$ provided by a thin superconducting solenoid. The CTD consists of 72 cylindrical drift chamber layers, organised in nine superlayers covering the polar-angle ${ }^{2}$ region $15^{\circ}<\theta<164^{\circ}(2.02>\eta>-1.96)$. The transverse-momentum resolution for full-length tracks is $\sigma\left(p_{T}\right) / p_{T}=0.0058 p_{T} \oplus 0.0065 \oplus 0.0014 / p_{T}$, with $p_{T}$ in $\mathrm{GeV}$.

The high-resolution uranium-scintillator calorimeter (CAL) [17] consists of three parts: the forward (FCAL, $1.1<\eta<3.8$ ), the barrel (BCAL, $-0.7<\eta<1.1$ ) and the rear (RCAL, $-3.4<\eta<-0.7$ ) calorimeters. Each part is subdivided transversely into towers and longitudinally into one electromagnetic section (EMC) and either one (RCAL) or two (BCAL and FCAL) hadronic sections (HAC). The smallest subdivision of the calorimeter is called a cell. In the EMC section, the towers are divided transversely into either four (FCAL and BCAL) or two (RCAL) cells. The CAL energy resolutions, as measured under test-beam conditions, are $\sigma(E) / E=0.18 / \sqrt{E}$ for electrons and $\sigma(E) / E=0.35 / \sqrt{E}$ for hadrons, with $E$ in $\mathrm{GeV}$.

Presampler detectors [18 are mounted in front of the CAL. They consist of scintillator tiles matching the calorimeter towers and measure signals from particle showers created by interactions in the material lying between the interaction point and the calorimeter. In this analysis, only the information from the presampler in front of the RCAL was used to correct the energy of the final-state particles.

The forward plug calorimeter (FPC) [19] is a lead-scintillator sandwich calorimeter with readout via wavelength-shifter fibres. It was installed in 1998 in the $20 \times 20 \mathrm{~cm}^{2}$ beam

\footnotetext{
${ }^{1}$ Hereafter, both $e^{+}$and $e^{-}$are referred to as electrons, unless explicitly stated otherwise.

${ }^{2}$ The ZEUS coordinate system is a right-handed Cartesian system, with the $Z$ axis pointing in the proton beam direction, referred to as the "forward direction", and the $X$ axis pointing left towards the centre of HERA. The coordinate origin is at the nominal interaction point. The pseudorapidity is defined as $\eta=-\ln \left(\tan \frac{\theta}{2}\right)$, where the polar angle, $\theta$, is measured with respect to the proton beam direction.
} 
hole of the FCAL and has a small hole of radius $3.15 \mathrm{~cm}$ in the centre to accommodate the beam pipe. It extends the pseudorapidity coverage of the forward calorimeter from $\eta<4.0$ to $\eta<5.0$. The FPC information was used to remove low-mass proton-dissociative events from the analysis.

The hadron-electron separator (HES) 20] is installed in the RCAL and FCAL. It consists of $3 \times 3 \mathrm{~cm}^{2}$ silicon diodes placed at a longitudinal depth of three radiation lengths, which corresponds to the approximate position of the maximum of the electromagnetic shower in the CAL. The separation between electrons and hadrons is based on the fact that the hadronic interaction length is 20 times larger than the electromagnetic radiation length. In this analysis, the fine segmentation of the RHES was used to improve the position resolution for both scattered electrons and photons.

The small-angle rear tracking detector (SRTD) [21] is attached to the front face of the $\operatorname{RCAL}(Z=-148 \mathrm{~cm})$. The SRTD consists of two planes of scintillator strips read out via optical fibres and photomultiplier tubes. It covers the region $68 \times 68 \mathrm{~cm}^{2}$ in $X$ and $Y$ with the exclusion of a $8 \times 20 \mathrm{~cm}^{2}$ hole at the centre for the beam pipe. The SRTD provides a transverse-position resolution of $3 \mathrm{~mm}$ and was used to measure the positions of photons and electrons scattered at small angles relative to the lepton beam direction.

The proton-remnant tagger (PRT1) [22] consists of two layers of scintillation counters located at $Z=5.15 \mathrm{~m}$, and covers the pseudorapidity range $4.3<\eta<5.8$. It was used, up to the end of the 1997 running period, to tag events in which the proton diffractively dissociated.

The luminosity was determined from the rate of the bremsstrahlung process $e p \rightarrow e \gamma p$, where the high-energy photon was measured with a lead-scintillator calorimeter [23] located at $Z=-107 \mathrm{~m}$.

\section{Event selection}

For the $Q^{2}$ range of this analysis, $Q^{2}>5 \mathrm{GeV}^{2}$, and small $t$, the signature of DVCS and $\mathrm{BH}$ events consists of a photon and a scattered electron with balanced transverse momenta. The scattered proton stays in the beam pipe and remains undetected.

The events were selected online via a three-level trigger system [15, 24. The trigger selected events with two isolated electromagnetic (EM) clusters in the EMC with energy greater than $2 \mathrm{GeV}$. The events were selected offline by requiring two EM clusters, the first in the RCAL with energy $E_{1}>15 \mathrm{GeV}$ and the second, with polar angle $0.6<\theta_{2}<$ $2.75 \mathrm{rad}\left(1.2>\eta_{2}>-1.6\right)$, either in the RCAL, with energy $E_{2}>3 \mathrm{GeV}$, or in the BCAL, with energy $E_{2}>2.5 \mathrm{GeV}$. The angular range of the second cluster corresponds to the 
region of high efficiency for reconstruction of a track in the CTD. If a track was found, it was required to match one of the EM clusters. Events with more than one track were rejected. To ensure full containment of the electromagnetic showers in the CAL, events in which one of the clusters was located within $3 \mathrm{~cm}$ of the beam hole were rejected.

The selection $40<E-p_{Z}<70 \mathrm{GeV}$ was imposed, where $E$ is the total energy and $p_{Z}$ the sum of $E \cos \theta$ over the whole CAL. This requirement rejects photoproduction events and events in which a hard photon is radiated from the incoming electron.

After these cuts, when the two EM clusters are ordered in energy such that $E_{1}>E_{2}$, the kinematics ensure $\eta_{1}<\eta_{2}$. In the following, the two clusters will be denoted as EM1 and EM2, respectively.

For the 96-97 (98-00) running period, calorimeter cells not associated with the two electromagnetic clusters were required to have energy less than: 150 (200) MeV in the FEMC and 200 (300) $\mathrm{MeV}$ in the FHAC; 200 (350) MeV in the BEMC and 250 (350) MeV in the BHAC; 150 (150) MeV in the REMC and 300 (300) MeV in the RHAC. These thresholds were set to be three standard deviations above the noise level of the CAL. Moreover, for the 98-00 data sample, the energy measured in the FPC was required to be less than $1 \mathrm{GeV}$. These elasticity requirements reject most events in which the proton dissociates into a hadronic system, $X$.

The events were subdivided into three samples, a first in which there was no track associated with the EM2 cluster ( $\gamma$ sample), a second in which the track associated with EM2 had the same charge as the beam electron ( $e$ sample) and finally a third in which the track associated with EM2 had the opposite charge to that of the beam electron (wrong-sign-e sample). These samples are interpreted as:

- $\gamma$ sample: EM2, with no track pointing to it, is the photon candidate and EM1 is the scattered-electron candidate. Both BH and DVCS processes contribute to this topology. The sample consisted of 3945 events.

- e sample: EM2, with the right-charge track pointing to it, is the scattered-electron candidate and EM1 is the photon candidate. This sample is dominated by the $\mathrm{BH}$ process. The contribution from DVCS is predicted to be negligible, due to the large $Q^{2}$ required for a large electron scattering angle. This sample contained 7059 events.

- wrong-sign- $e$ sample: EM2, with the wrong-charge-sign track pointing to it, may have originated from an $e^{+} e^{-}$final state accompanying the scattered electron, where one of the right-sign electrons escaped detection. This background sample is due to nonresonant $e^{+} e^{-}$production and to $J / \psi$ production and subsequent decay. Other sources are negligible, as will be discussed later. This sample consisted of 287 events.

The wrong-sign-e sample was used to statistically subtract the background contributions 
to the $e$ sample in each kinematic bin. The background-subtracted $e$ sample was then used to investigate the $\mathrm{BH}$ contribution to the $\gamma$ sample.

For the purposes of this analysis, the values of $Q^{2}$ and $W$ were determined for each event, independently of its topology, under the assumption that the EM1 cluster is the scattered electron. This assumption is always valid for DVCS events for the $Q^{2}$ range considered here. The value of $Q^{2}$ was calculated using the electron method [25], while $W$ was determined using the double-angle method [25]. No explicit cut on $t$ was applied

in the event selection. Events for which $40<W<140 \mathrm{GeV}$ and $5<Q^{2}<100 \mathrm{GeV}^{2}$ were retained.

\section{Monte Carlo simulations}

The acceptance and the detector response were determined using Monte Carlo (MC) simulations. The detector was simulated in detail using a program based on GEANT 3.13 [26]. All of the simulated events were processed through the same reconstruction and analysis chain as the data.

A MC generator, GenDVCS [27] based on a model by Frankfurt, Freund and Strikman (FFS) 4], was used to simulate the elastic DVCS process. In the FFS calculation, the DVCS cross section, integrated over the angle between the $e$ and $p$ scattering planes, is related to the inclusive structure-function $F_{2}$ through

$$
\frac{d^{3} \sigma_{\mathrm{DVCS}}^{e p \rightarrow e \gamma p}}{d x d Q^{2} d t}=\frac{\pi^{2} \alpha^{3}}{2 x R^{2} Q^{6}}\left[1+(1-y)^{2}\right] e^{-b|t|} F_{2}^{2}\left(x, Q^{2}\right)\left(1+\rho^{2}\right),
$$

where $x \simeq Q^{2} /\left(Q^{2}+W^{2}\right)$ is the Bjorken scaling variable, $b$ is the exponential slope of the $t$ dependence and $y$ is the fraction of the electron energy transferred to the proton in its rest frame. The ratio $R=\left.\Im m \mathcal{A}\left(\gamma^{*} p \rightarrow \gamma^{*} p\right)\right|_{t=0} /\left.\Im m \mathcal{A}\left(\gamma^{*} p \rightarrow \gamma p\right)\right|_{t=0}$ accounts for the non-forward character of the DVCS process and is directly related to a ratio of the GPD to the parton distribution functions [28] and $\rho$ is the ratio of the real to imaginary part of the amplitude, $\rho=\left.\Re e \mathcal{A}\left(\gamma^{*} p \rightarrow \gamma p\right)\right|_{t=0} /\left.\Im m \mathcal{A}\left(\gamma^{*} p \rightarrow \gamma p\right)\right|_{t=0}$.

The value of $R$, calculated using the leading-order (LO) QCD evolution of the GPD, is about 0.55 , with little dependence on $x$ or $Q^{2}$ [4]. For simplicity, the $R$ parameter in the $\mathrm{MC}$ generator was set to a constant value of $R=0.55$.

In GenDVCS, the ALLM97 [29] parameterisation of the $F_{2}$ structure function of the proton was used as input. In this empirical fit to $\gamma^{*} p$ total-cross-section data, the value of $\rho$ was parameterised as $\rho=\frac{\pi}{2}\left(0.176+0.033 \ln Q^{2}\right)$, where $Q^{2}$ is in $\mathrm{GeV}^{2}$ [27].

In the FFS model, the $t$ dependence is assumed to factorise, with the slope parameter, $b$, depending on both $W$ and $Q^{2}$. The value of $b$ is expected to decrease with $Q^{2}$ and, even 
at high $Q^{2}$ and at very small $x$, is expected to increase with $W$. While this dependence is important for the normalisation of the calculated DVCS cross section, it does not affect the acceptance corrections. In the MC simulation $b$ was assumed to be constant and was set to $4.5 \mathrm{GeV}^{-2}$.

For proper treatment of radiative effects, the GenDVCS generator was interfaced to HERACLES 4.6 [30], which includes corrections for initial- and final-state photon emission from the electron line, as well as vertex and propagator corrections.

The elastic and inelastic BH processes, ep $\rightarrow e \gamma p$ and $e p \rightarrow e \gamma X$, and the QED dilepton production, $e p \rightarrow e e^{+} e^{-} p$, were simulated using the GRAPE-Compton ${ }^{3}$ [31 and GRAPE-Dilepton [31] generators, respectively. These two MC programs are based on the automatic system GRACE [32] for calculating Feynman diagrams. The GRAPE generator gave identical results to the Compton 2.0 33] generator for the elastic BH process. The GRAPE program was used because it simulates the hadronic final state for the inelastic $\mathrm{BH}$ process.

Additional samples were generated using the diffractive RAPGAP [34] and non-diffractive DJANGOH [35] generators in order to study possible backgrounds from low-multiplicity DIS events. A possible contribution from vector-meson electroproduction was simulated by the ZEUSVM [36] MC generator interfaced to HERACLES.

\section{$5 \quad$ DVCS-signal extraction}

In the kinematic region of this analysis the interference between the DVCS and BH amplitudes is very small when the cross section is integrated over the angle between the $e$ and $p$ scattering planes [8, 9]. Thus the cross section for exclusive production of real photons may be treated as a simple sum over the contributions from the DVCS and electromagnetic $\mathrm{BH}$ processes. The latter can, therefore, be subtracted and the DVCS cross section determined.

The $\mathrm{BH}$ process was studied using the $e$ sample which, according to the MC predictions, consists almost solely of $\mathrm{BH}$ candidates. A background contribution to the $\mathrm{BH}$ events in this sample of about $4 \%$, originating from deep inelastic exclusive $e^{+} e^{-}$production, where one of three final-state leptons escapes detection, was estimated from the wrong-sign-e sample and MC simulations, and was statistically subtracted. According to the MC simulations, $75 \%$ of this background consists of non-resonant di-lepton production and $25 \%$ of exclusive $J / \psi$ production with subsequent $e^{+} e^{-}$decay. The normalisation of

\footnotetext{
${ }^{3}$ Hereafter, the GRAPE-Compton generator is referred as GRAPE
} 
the MC samples was determined from the wrong-sign- $e$ sample. The diffractive electroproduction of $\rho, \omega$ and $\phi$ mesons, in which one of the decay charged particles was misidentified as an electron in the CAL, the other was undetected, and the electron scattered into the RCAL was taken to be the photon, was negligible.

The expectation for the inelastic $\mathrm{BH}$ contribution to the $e$ sample is subject to uncertainties coming from the dependence of the selection efficiency on the mass of the hadronic final-state system, $X$. This inelastic contribution was estimated from the data as $(17.8 \pm 1.2) \%$, for the $96-97$ data sample, using the fraction of events tagged in the PRT1 and (10.5 \pm 1.0$) \%$, for the 98-00 data sample, using events with more than $1 \mathrm{GeV}$ of energy in the FPC, obtained releasing the elasticity cut. The uncertainties are statistical. No attempt was made to quantify the systematic uncertainty since there is little sensitivity to the cross section. The difference in the measured fraction of proton-dissociative events is due to differences in the detector configuration between the two running periods. After the subtraction of the dilepton and $J / \psi$ backgrounds, for the 96-97 (99-00) $e^{+} p$ samples, the number of remaining BH events in the $e$ sample was 2523 (3289), while the expected number from the GRAPE simulation was 2601 (3358). The absolute expectation of the GRAPE simulation reproduced the number of BH data events to within $(3 \pm 5) \%$ for 96-97 and (2 \pm 4$) \%$ for 99-00, where uncertainties include the statistical uncertainty as well as the uncertainties due to the trigger efficiency and the estimation of the inelastic $\mathrm{BH}$ contribution.

For further analysis, the GRAPE MC sample was normalised to the BH events in the data. The comparison between the $e$ sample and the sum of the GRAPE BH, dilepton and $J / \psi$ MC samples for the 99-00 $e^{+} p$ running period is shown in Fig. 2a versus $W$ and in Fig. 2 2 versus the difference in azimuthal angles of the EM1 and the EM2, $\Delta \phi_{12}$. Good agreement is observed.

The properties of the $\gamma$ sample were then compared to the expectations of the normalised GRAPE MC sample. As an example, the comparison of the $W$ and $\Delta \phi_{12}$ distributions for the 99-00 $e^{+} p$ running period is shown in Figs. 22 and 2 $\mathrm{d}$, respectively. An excess of events over the expectations of the GRAPE simulation is observed. Moreover, the data distributions differ from those expected for the $\mathrm{BH}$ process. The $W$ distribution of the $\mathrm{BH}$ sample peaks at large $W$, while that of the data is more evenly distributed. The $\Delta \phi_{12}$ distribution of the $\mathrm{BH}$ sample is also narrower than that of the data.

The $W$ and the $\Delta \phi_{12}$ distributions of the data, after subtracting the $\mathrm{BH}$ contribution using the renormalised GRAPE MC sample, are shown in Figs. 25 and 2;, respectively. This sample includes events in which the proton dissociated into a hadronic final state with low mass.

The previous ZEUS measurements of elastic vector meson production [37, 38] support, 
within relatively large uncertainties, the assumption that the fraction of proton dissociative events, $f_{\mathrm{p}-\text { diss }}$, in diffractive interaction is process independent. Therefore, in this analysis, the values of $f_{\mathrm{p} \text {-diss }}$ determined from the measurements of the diffractive $\mathrm{J} / \mathrm{psi}$ photoproduction 38, are used

$$
\begin{array}{lll}
f_{\mathrm{p}-\text { diss }}=22.0 \pm 2.0(\text { stat. }) \pm 2.0 \text { (syst.) } \% & \text { for the } 96-97 \text { data; } \\
\left.f_{\mathrm{p}-\text { diss }}=17.5 \pm 1.3 \text { (stat. }\right)_{-3.2}^{+3.7} \text { (syst.) } \% & \text { for the 98-00 data. }
\end{array}
$$

The above fractions are consistent, within large uncertainties, with those estimated using the events in the $\gamma$ sample either tagged by the PRT1 or the FPC, after subtracting the inelastic BH contributions.

Other possible sources of contamination were investigated. Due to the relatively high $Q^{2}$ of the present data set, the contamination from production of light vector mesons, such as $\omega$ or $\phi$, decaying through channels containing photons in the final state is below $1 \%$ and was neglected. A possible contribution to the $\gamma$ sample from low-multiplicity processes such as $e p \rightarrow e \pi^{0} p$, ep $\rightarrow e \pi^{0} \pi^{0} p$, and $e p \rightarrow e \pi^{0} \eta p$, where the $\pi^{0}$ or $\eta$ fakes a photon signal, was also investigated. The number of candidate events found in the RAPGAP and DJANGOH samples was reweighted to reflect the cross sections obtained by extrapolating low- $W$ measurements [39, 40]. Their contribution to the DVCS sample is negligible.

The data are compared to the absolute expectations of GenDVCS in Figs. 20 and 2f: The best agreement in normalisation between the data and the MC simulation is achieved when the normalisation of the latter is decreased by $10 \%$. This was obtained by increasing the value of $b$ from 4.5 to $4.9 \mathrm{GeV}^{-2}$ (see Section 4). Overall, good agreement between the data and the $\mathrm{MC}$ simulation is found, demonstrating that the excess of photon candidates over the expectation of $\mathrm{BH}$ is due to DVCS.

\section{Cross-section determination}

The $\gamma^{*} p$ cross section for the DVCS process as a function of $W$ and $Q^{2}$ was evaluated using the expression

$$
\sigma\left(\gamma^{*} p \rightarrow \gamma p\right)\left(W_{i}, Q_{i}^{2}\right)=\frac{\left(N_{i}^{\mathrm{obs}}-N_{i}^{\mathrm{BH}}\right) \cdot\left(1-f_{\mathrm{p}-\mathrm{diss}}\right)}{N_{i}^{\mathrm{MC}}} \cdot \sigma^{\mathrm{FFS}}\left(\gamma^{*} p \rightarrow \gamma p\right)\left(W_{i}, Q_{i}^{2}\right)
$$

where $N_{i}^{\text {obs }}$ is the total number of data events in the $\gamma$ sample in bin $i$ in $W$ and $Q^{2}$, $N_{i}^{\mathrm{BH}}$ denotes the number of $\mathrm{BH}$ events in the $\gamma$ sample in that bin, determined from the renormalised GRAPE sample, and $N_{i}^{\mathrm{MC}}$ is the number of events expected in the $\gamma$ sample from GenDVCS for the luminosity of the data. The factor $f_{\mathrm{p}-\text { diss }}$ is the fraction of the

proton-dissociative DVCS events in the data, $\sigma^{\mathrm{FFS}}\left(\gamma^{*} p \rightarrow \gamma p\right)$ is the $\gamma^{*} p$ cross section 
computed according to the FFS expression, and $W_{i}$ and $Q_{i}^{2}$ are the values of $W$ and $Q^{2}$ where the cross section is evaluated.

The $\gamma^{*} p$ cross sections have been computed in the ranges $5<Q^{2}<100 \mathrm{GeV}^{2}$ and $40<$ $W<140 \mathrm{GeV}$, separately for the 96-97, 98-99 and 99-00 data periods and then combined for the positron samples (96-97 and 99-00). Tables 1 - 3 list the $\gamma^{*} p \rightarrow \gamma p$ cross-section values.

\section{$7 \quad$ Systematic uncertainties}

The systematic uncertainties of the measured cross sections were determined by changing the selection cuts or the analysis procedure in turn and repeating the extraction of the cross sections. The following systematic studies have been carried out:

- all the selection cuts discussed in Section 3 were shifted according to the resolutions of the corresponding variables. The most significant contributions came from varying the lower $Q^{2}$ cut. The average change in the cross section due to this cut was $\pm 2 \%$. The largest change in the cross section, $\pm 10 \%$, was found in the highest- $W$ bin, while it was $\pm 4 \%$ in the lowest- $Q^{2}$ bin;

- the elasticity cut was changed by $\pm 30 \mathrm{MeV}$ in the EMC and $\pm 50 \mathrm{MeV}$ in the HAC. The average change in the cross section was $\pm 2 \%$ in all bins of $Q^{2}$ and $W$, while the largest change in the cross section, observed when the cut was lowered, was $-4 \%$ in the lowest- $W$ bins and $-4 \%$ in the lowest- $Q^{2}$ bin;

- the trigger efficiency was varied within its statistical uncertainty. This resulted in average changes of the cross section of about $\pm 2 \%$. The biggest variation of the cross section of $\pm 3 \%$ was observed in the lowest- $Q^{2}$ bin and in the two highest- $W$ bins;

- the electromagnetic energy scale was varied within its uncertainty of $1.5 \%$ for the EM2 (low energy) and of $1 \%$ for the EM1 (high energy), resulting in a $\pm 3 \%$ average change of the cross section in both $Q^{2}$ and $W$. The largest change was $\pm 3 \%$ for the lowest- $Q^{2}$ bins and $\pm 5 \%$ for the highest- $W$ bin;

- in GenDVCS, the $Q^{2}$ dependence was modified by introducing a $Q^{2}$-dependent $t$ slope using the formula $b=8\left(1-0.15 \ln \left(Q^{2} / 2\right)\right) \mathrm{GeV}^{-2}$ (see Section 9). The average change in the cross section was $\pm 1 \%$, with the largest variation of $\pm 3 \%$ in the highest- $Q^{2}$ bin.

The uncertainty on the proton-dissociative contribution, $f_{\mathrm{p} \text {-diss }}$, leads to an overall normalisation uncertainty of $\pm 4.0 \%$ and $\pm 3.5 \%$ for the $e^{-} p$ and $e^{+} p$ data, respectively.

The systematic uncertainties typically are small compared to the statistical uncertainties. The individual systematic uncertainties, including that due to $f_{\mathrm{p} \text {-diss }}$, were added in 
quadrature separately for the positive and negative deviations from the nominal crosssection values to obtain the total systematic uncertainties listed in Tables 1- 3. An overall normalisation uncertainty in the luminosity determination of $\pm 1.8 \%$ and $\pm 2.0 \%$ for the $e^{-} p$ and $e^{+} p$ data, respectively, was not included because it was small with respect to the above contributions.

\section{Results}

The $W$ dependence of the DVCS cross section, $\sigma_{\text {DVCS }}=\sigma\left(\gamma^{*} p \rightarrow \gamma p\right)$, for $Q^{2}=9.6 \mathrm{GeV}^{2}$ is shown in Fig. 3, separately for $e^{+} p$ and $e^{-} p$ interactions. Due to the limited statistics, the $e^{-}$sample is only shown in three $W$ bins. There is agreement between the two samples. A fit of the form $\sigma_{\text {DVCS }} \propto W^{\delta}$ was performed separately for the positron and electron data. For the $e^{+} p$ data, the value $\delta=0.75 \pm 0.15$ (stat.) ${ }_{-0.06}^{+0.08}$ (syst.) is comparable to that determined for $J / \psi$ electroproduction [38. This steep rise in the cross section is a strong indication of the presence of a hard underlying process. The same fit to the $e^{-} p$ data yields $\delta=0.45 \pm 0.36$ (stat.) ${ }_{-0.07}^{+0.08}$ (syst.), which is compatible with the $e^{+} p$ result.

The positron sample has been further subdivided into three $Q^{2}$ ranges. The $W$ dependence of $\sigma_{\text {DVCS }}$ in these three $Q^{2}$ bins is presented in Fig. 4. The results are compatible with no dependence of $\delta$ on $Q^{2}$ although also with the increase with $Q^{2}$ observed in exclusive production of light vector mesons [41,37.

The $Q^{2}$ dependence of $\sigma_{\text {DVCS }}$, for $W=89 \mathrm{GeV}$, is shown in Fig. 5 a, again separately for $e^{+} p$ and $e^{-} p$ interactions. There is no significant cross-section difference between the $e^{+}$ and $e^{-}$data, which is consistent with the assumption that the present measurement is insensitive to the interference term.

A fit of the form $Q^{-2 n}$ to the $e^{+} p$ data gives a value of $n=1.54 \pm 0.07$ (stat.) \pm 0.06 (syst.). This value is lower than $n \simeq 2$ which is characteristic of exclusive vector-meson production [37, 42]. The fit to the $e^{-} p$ data gives $n=1.69 \pm 0.21$ (stat.) ${ }_{-0.06}^{+0.09}$ (syst.).

\section{Comparison with models}

In the presence of a hard scale $\left(Q^{2}>>\Lambda_{\mathrm{QCD}}^{2}\right)$, the DVCS amplitude factorises into a hard-scattering coefficient, calculable in pQCD, and a soft part which can be absorbed in the GPD [3]. The kernels of the evolution equations for the GPD are known to nextto-leading order (NLO) [43, 44] and the GPD can thus be evaluated at all $Q^{2}$ given an input at some starting scale. Measurements of the DVCS cross section are an essential ingredient in modelling the input GPD [44,28]. 
Freund, McDermott and Strikman (FMS) [28] have made an attempt to model the GPD based on DVCS data [12, 13, 14]. A comparison of $\sigma_{\text {DVCS }}$ as a function of $Q^{2}$ for fixed $W$ with the predictions based on the MRST parameterisation of the parton distribution functions (PDF) 45, 46] is shown in Fig. [5. Three FMS curves are shown. Two curves show the results of modelling based on LO (MRSTL) and NLO (MRSTM) parton distribution functions. The latter leads to predictions closer to the data. In this comparison, a fixed value of the $t$ slope, $b=4.9 \mathrm{GeV}^{-2}$, was assumed. The third curve, shown in the figure, corresponds to predictions based on MRSTM, assuming a $Q^{2}$-dependent $b$ value. The best agreement between the data and the predictions is achieved using $b=8\left(1-0.15 \ln \left(Q^{2} / 2\right)\right) \mathrm{GeV}^{-2}$, a parameterisation obtained by Freund, McDermott and Strikman [28] from a fit to a preliminary version of the present data. Similar conclusions are reached when the CTEQ6 parameterisations [47,48,49] are used (not shown).

The data are also compared to the expectations of FFS (see Section 4), again assuming $b=4.9 \mathrm{GeV}^{-2}$. For $Q^{2}>20 \mathrm{GeV}^{2}$, the $e^{+} p$ data lie significantly above the prediction.

The DVCS cross section has also been calculated within colour-dipole models [50, 51, 52, 53, 54, which have been successful in describing both the inclusive and the diffractive DIS cross sections at high energy [55, 56, 57, 58, 59, 60]. The various dipole models differ in their formulation of the dipole cross section with the target proton. If $s$-channel helicity is conserved in DVCS, the virtual photon must be transversely polarised. As the wave function of the transversely-polarised photon can select large dipole sizes, whose interactions are predominantly soft, DVCS constitutes a good probe of the transition between perturbative and non-perturbative regimes of QCD. The $Q^{2}$ dependence of $\sigma_{\text {DVCS }}$ has been compared to the expectations of three calculations based on colour-dipole models, by Donnachie and Dosch (DD) [60, Forshaw, Kerley and Shaw (FKS) [57, 61, 62] and McDermott, Frankfurt, Guzey and Strikman (MFGS) 62,59. The comparisons are shown

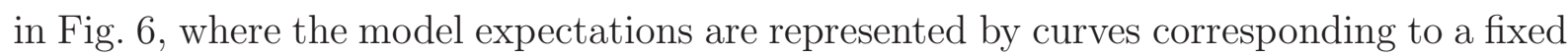
value of $b=4 \mathrm{GeV}^{-2}$ (upper) and $b=7 \mathrm{GeV}^{-2}$ (lower), chosen for illustration. All three predictions give a reasonable representation of the data. The $\mathrm{H} 1$ measurements 12 are also shown, extrapolated to the $W$ value of the ZEUS data using the $W^{\delta}$ dependence of the cross section measured in this analysis for the $e^{+} p$ data. The H1 data lie systematically below the ZEUS data.

\section{Conclusions}

The DVCS cross section $\sigma\left(\gamma^{*} p \rightarrow \gamma p\right)$ has been measured at HERA in the kinematic range $5<Q^{2}<100 \mathrm{GeV}^{2}$ and $40<W<140 \mathrm{GeV}$. No significant difference between the $e^{+} p$ and $e^{-} p$ interactions was observed. The data have been compared to calculations 
based on generalised parton distributions and on the colour-dipole model. Generally, good agreement with the data is observed.

The $Q^{2}$ dependence of the DVCS cross section follows approximately a $Q^{-3}$ behaviour. The precision of the data allows an accurate determination of the $W$ distribution for the first time. The cross section rises steeply with $W$, indicative of a hard underlying process, where the rise reflects the increase of parton distributions with decreasing Bjorken $x$.

These measurements demonstrate the potential of DVCS data to constrain the structure of the proton and quark-gluon dynamics at low $x$.

\section{Acknowledgements}

We thank the DESY directorate for their strong support and encouragement. The special efforts of the HERA machine group are gratefully acknowledged. We are grateful for the support of the DESY computing and network services. The design, construction and installation of the ZEUS detector have been made possible by the ingenuity and effort of many people who are not listed as authors. We are grateful to M. Diehl, A. Freund, M. McDermott and M. Strikman for numerous discussions in which they offered invaluable insight into the DVCS process. We thank M. McDermott and R. Sandapen for providing the model calculations.

We dedicate this paper to the memory of Bill Toothacker whose early work on the analysis of DVCS helped us to obtain the preliminary results that were shown at the EPS meeting in Tampere, Finland, July 1999. 


\section{References}

[1] E. Wieczorek, V.A. Matveev and D. Robaschik, Theoret. Mat. Phys. 19, 315 (1974);

J. Bartels and M. Loewe, Z. Phys. C 12, 263 (1982);

X. Ji, Phys. Rev. D 55, 7114 (1997).

[2] A.V. Radyushkin, Phys. Rev. D 58, 114008 (1998).

[3] J.C. Collins and A. Freund, Phys. Rev. D 59, 74009 (1999).

[4] L.L. Frankfurt, A. Freund and M. Strikman, Phys. Rev. D 58, 114001 (1998). Erratum ibid. D 59119901 (1999).

[5] D. Müller et al., Fortschr. Phys. 42, 347 (1994).

[6] X. Ji, Phys. Rev. Lett. 78, 610 (1997).

[7] K. Golec-Biernat and A. Martin, Phys. Rev. D 59, 014029 (1999).

[8] L. Frankfurt, A. Freund and M. Strikman, Phys. Lett. B 460, 417 (1999).

[9] A.V. Belitsky, D. Müller and A. Kirchner, Nucl. Phys. B 629, 323 (2002).

[10] A. Freund and M. McDermott, Phys. Rev. D65, 091901 (2002).

[11] P.R.B. Saull, Proc. of the International Europhysics Conference on High-Energy Physics (EPS-HEP 99), p. 420-422, Tampere, Finland, 15-21 July 1999, (hep-ex/0003030).

[12] H1 Coll., C. Adloff et al., Phys. Lett. B 517, 47 (2001).

[13] HERMES Coll., A. Airapetian et al., Phys. Rev. Lett. 87, 182001 (2001).

[14] CLAS Coll., S. Stepanyan et al., Phys. Rev. Lett. 87, 182002 (2001).

[15] ZEUS Coll., U. Holm (ed.), The ZEUS Detector. Status Report (unpublished), DESY (1993), available on http://www-zeus.desy.de/bluebook/bluebook.html.

[16] N. Harnew et al., Nucl. Inst. Meth. A 279, 290 (1989);

B. Foster et al., Nucl. Phys. Proc. Suppl. B 32, 181 (1993);

B. Foster et al., Nucl. Inst. Meth. A 338, 254 (1994).

[17] M. Derrick et al., Nucl. Inst. Meth. A 309, 77 (1991);

A. Andresen et al., Nucl. Inst. Meth. A 309, 101 (1991);

A. Caldwell et al., Nucl. Inst. Meth. A 321, 356 (1992);

A. Bernstein et al., Nucl. Inst. Meth. A 336, 23 (1993).

[18] A. Bamberger et al., Nucl. Inst. Meth. A 382, 419 (1996);

S. Magill and S. Chekanov, Proc. of the IX International Conference on Calorimetry in High Energy Physics, B. Aubert et al. (eds.), p. 625, Annecy, France (2000). 
[19] ZEUS Coll., FPC group, A. Bamberger et al., Nucl. Inst. Meth. A 450, 235 (2000).

[20] A. Dwurazny et al., Nucl. Inst. Meth. A 277, 176 (1989).

[21] A. Bamberger et al., Nucl. Inst. Meth. A 401, 63 (1997).

[22] ZEUS Coll., J. Breitweg et al., Z. Phys. C 75, 421 (1997).

[23] J. Andruszków et al., Preprint DESY-92-066, DESY, 1992;

ZEUS Coll., M. Derrick et al., Z. Phys. C 63, 391 (1994);

J. Andruszków et al., Acta Phys. Pol. B 32, 2025 (2001).

[24] W.H. Smith, K. Tokushuku and L.W. Wiggers, Proc. Computing in High-Energy Physics (CHEP), Annecy, France, Sept. 1992, C. Verkerk and W. Wojcik (eds.), p. 222, CERN, Geneva, Switzerland (1992). Also in preprint DESY 92-150B.

[25] S. Bentvelsen, J. Engelen and P. Kooijman, Proc. Workshop on Physics at HERA, W. Buchmüller and G. Ingelman (eds.), Vol. 1, p. 23, Hamburg, Germany, DESY (1992);

K.C. Höger, ibid, p.43.

[26] R. Brun et al., GEANT3. Technical Report CERN-DD/EE/84-1,CERN, 1987.

[27] P.R.B. Saull, A Monte Carlo Generator for Deeply Virtual Compton Scattering at HERA, 1999, available on http://www-zeus.desy.de/physics/diff/pub/MC.

[28] A. Freund, M. McDermott and M. Strikman, Phys. Rev. D 67, 036001 (2003).

[29] H. Abramowicz and A. Levy, Preprint DESY-97-251 (hep-ph/9712415), DESY, 1997.

[30] H. Spiesberger, An Event Generator for ep Interactions at HERA Including Radiative Processes (Version 4.6), 1996, available on http://www.desy.de/ hspiesb/heracles.html.

[31] T. Abe, Comp. Phys. Comm. 136, 126 (2001).

[32] T. Ishikawa et al., GRACE manual: Automatic generation of tree amplitudes in Standard Models: Version 1.0. KEK Report 92-19, 1993.

[33] T. Carli et al., Proc. Workshop on Physics at HERA, W. Buchmüller and G. Ingelman (eds.), Vol. 3, p. 1468, Hamurg, Germany, DESY (1992).

[34] H. Jung, Comp. Phys. Comm. 86, 147 (1995).

[35] H. Spiesberger, HERACLES and DJANGOH: Event Generation for ep Interactions at HERA Including Radiative Processes, 1998, available on http://www.desy.de/ ${ }^{\sim}$ hspiesb/djangoh.html.

[36] K. Muchorowski, Ph.D. Thesis, Warsaw University, 1998 (unpublished). 
[37] ZEUS Coll., J. Breitweg et al., Eur. Phys. J. C 6, 603 (1999);

ZEUS Coll., J. Breitweg et al., Eur. Phys. J. C 14, 213 (2000).

[38] ZEUS Coll., S. Chekanov et al., Eur. Phys. J. C 24, 345 (2002).

[39] R. L. Anderson et al., Phys. Rev. D 7, 1937 (1971).

[40] S. I. Alekhin et al., CERN-HERA 87-01, 1987.

[41] H1 Coll., C. Adloff et al., Eur. Phys. J. C 13, 371 (2000).

[42] H1 Coll., C. Adloff et al., Phys. Lett. B 483, 360 (2000).

[43] A. V. Belitsky, D. Müller and A. Freund, Phys. Lett. B 461, 270 (1999).

[44] A. Freund and M. McDermott, Eur. Phys. J. C 23, 651 (2002);

A. Freund and M. McDermott, Phys. Rev. D 65, 091901 (2002).

[45] R.S. Thorne et al., Proc. of the 9th International Workshop on Deep Inelastic Scattering (DIS 2001), G. Bruni, G. Iacobucci and R. Nania (eds.), p. 405-408, Bologna, Italy, 27 Apr-1 May 2001, (hep-ph/0106075).

[46] A.D. Martin et al., Nucl. Phys. Proc. Suppl. 79, 105 (1999).

[47] H.L. Lai et al., Phys. Rev. D 51, 4763 (1995).

[48] J. Botts et al., Phys. Lett. B 304, 159 (1995).

[49] CTEQ Coll., H.L. Lai et al., Eur. Phys. J. C 12, 375 (2000).

[50] N.N. Nikolaev and B.G. Zakharov, Z. Phys. C 49, 607 (1991).

[51] N.N. Nikolaev and B.G. Zakharov, Z. Phys. C 53, 331 (1992).

[52] A.H. Mueller, Nucl. Phys. B 415, 373 (1994).

[53] A.H. Mueller and B. Patel, Nucl. Phys. B 425, 471 (1994).

[54] L. Favart and M.V.T. Machado, Preprint hep-ph/0302079, 2003.

[55] K. Golec-Biernat and M. Wüsthoff, Phys. Rev. D 59, 014017 (1999).

[56] K. Golec-Biernat and M. Wüsthoff, Phys. Rev. D 60, 114023 (1999).

[57] J.R. Forshaw, G. Kerley and G. Shaw, Phys. Rev. D 60, 074012 (1999).

[58] E. Gotsman, E. Levin and U. Maor, Phys. Lett. B 425, 369 (1998).

[59] M. McDermott et al., Eur. Phys. J. C 16, 641 (2000).

[60] A. Donnachie and H.G. Dosch, Phys. Lett. B 502, 74 (2001).

[61] J.R. Forshaw, G. Kerley and G. Shaw, Nucl. Phys. A 675, 80c (2000).

[62] M. McDermott, R. Sandapen and G. Shaw, Eur. Phys. J. C 22, 655 (2002). 


\begin{tabular}{|c|c|c|c|}
\hline \multicolumn{4}{|c|}{$\sigma^{\gamma^{*} p \rightarrow \gamma p}$} \\
\hline$Q^{2}$ range $\left(\mathrm{GeV}^{2}\right)$ & $Q^{2}\left(\mathrm{GeV}^{2}\right)$ & $\begin{array}{c}\sigma^{\gamma^{*} p \rightarrow \gamma p}(\mathrm{nb}) \\
e^{+} p\end{array}$ & $\begin{array}{c}\gamma^{\gamma^{*} p \rightarrow \gamma p}(\mathrm{nb}) \\
e^{-} p\end{array}$ \\
\hline $5-10$ & 7.5 & $5.42 \pm 0.33_{-0.34}^{+0.29}$ & $5.63 \pm 0.77_{-0.33}^{+0.30}$ \\
$10-15$ & 12.5 & $2.64 \pm 0.22_{-0.13}^{+0.11}$ & $2.20 \pm 0.52_{-0.14}^{+0.13}$ \\
$15-25$ & 20 & $1.23 \pm 0.14_{-0.07}^{+0.05}$ & $0.96 \pm 0.31_{-0.06}^{+0.10}$ \\
$25-40$ & 32.5 & $0.59 \pm 0.12_{-0.04}^{+0.04}$ & $0.61 \pm 0.28_{-0.05}^{+0.06}$ \\
$40-70$ & 55 & $0.20 \pm 0.08_{-0.02}^{+0.03}$ & - \\
$70-100$ & 85 & $0.16 \pm 0.09_{-0.03}^{+0.02}$ & - \\
\hline
\end{tabular}

Table 1: Values of the cross sections for the $\gamma^{*} p \rightarrow \gamma p$ DVCS process as a function of $Q^{2}$ for the $e^{+} p$ and $e^{-} p$ data. Values are quoted at the centre of each $Q^{2}$ bin and for the average $W$ value of the whole sample, $W=89 \mathrm{GeV}$, obtained from GenDVCS. The first uncertainty is statistical and the second systematic. The systematic uncertainty due to the luminosity determination is not included.

\begin{tabular}{|c|c|c|c|c|c|}
\hline \multicolumn{7}{|c|}{$\sigma^{\gamma^{*} p \rightarrow \gamma p}$} \\
\hline $\begin{array}{c}\text { range }(\mathrm{GeV}) \\
e^{+} p\end{array}$ & $\begin{array}{c}W(\mathrm{GeV}) \\
e^{+} p\end{array}$ & $\begin{array}{c}\sigma^{\gamma^{*} p \rightarrow \gamma p}(\mathrm{nb}) \\
e^{+} p\end{array}$ & $\begin{array}{c}W \text { range }(\mathrm{GeV}) \\
e^{-} p\end{array}$ & $\begin{array}{c}W(\mathrm{GeV}) \\
e^{-} p\end{array}$ & $\begin{array}{c}\sigma^{\gamma^{*} p \rightarrow \gamma p}(\mathrm{nb}) \\
e^{-} p\end{array}$ \\
\hline $40-50$ & 45 & $2.19 \pm 0.24_{-0.14}^{+0.11}$ & & & \\
$50-60$ & 55 & $2.96 \pm 0.28_{-0.18}^{+0.13}$ & & & \\
$60-70$ & 65 & $3.62 \pm 0.36_{-0.23}^{+0.18}$ & $40-73$ & 56.7 & $2.94 \pm 0.39_{-0.13}^{+0.16}$ \\
$70-80$ & 75 & $3.88 \pm 0.42_{-0.26}^{+0.18}$ & & \\
$80-90$ & 85 & $3.59 \pm 0.45_{-0.25}^{+0.18}$ & & \\
$90-100$ & 95 & $3.29 \pm 0.55_{-0.20}^{+0.21}$ & $73-107$ & & \\
$100-110$ & 105 & $6.24 \pm 0.77_{-0.49}^{+0.31}$ & & & \\
$110-120$ & 115 & $4.86 \pm 0.76_{-0.44}^{+0.39}$ & & & \\
$120-130$ & 125 & $4.69 \pm 0.82_{-0.36}^{+0.32}$ & $107-140$ & 123.3 & $3.8 \pm 1.1_{-0.4}^{+0.3}$ \\
$130-140$ & 135 & $5.55 \pm 0.99_{-0.30}^{+0.91}$ & & & \\
\hline
\end{tabular}

Table 2: Values of the cross sections for the $\gamma^{*} p \rightarrow \gamma p$ DVCS process as a function of $W$ for the $e^{+} p$ and $e^{-} p$ data. Values are quoted at the centre of each $W$ bin and for the average $Q^{2}$ value of the whole sample, $Q^{2}=9.6 \mathrm{GeV}^{2}$, obtained from GenDVCS. The first uncertainty is statistical and the second systematic. The systematic uncertainty due to the luminosity determination is not included. 


\begin{tabular}{|c|c|c|c|c|}
\hline \multicolumn{5}{|c|}{$\sigma^{\gamma^{*} p \rightarrow \gamma p}$} \\
\hline$W$ range $(\mathrm{GeV})$ & $W(\mathrm{GeV})$ & $\begin{array}{c}\sigma^{\gamma^{*} p \rightarrow \gamma p}(\mathrm{nb}) \\
5<Q^{2}<8 \mathrm{GeV}^{2} \\
\end{array}$ & $\begin{array}{c}\sigma^{\gamma^{*} p \rightarrow \gamma p}(\mathrm{nb}) \\
8<Q^{2}<13 \mathrm{GeV}^{2} \\
Q^{2}=6.2 \mathrm{GeV}^{2}\end{array}$ & $\begin{array}{c}\sigma^{\gamma^{*} p \rightarrow \gamma p}(\mathrm{nb}) \\
Q^{2}=9.9 \mathrm{GeV}^{2}\end{array}$ \\
\hline & & $\begin{array}{c}2 \\
Q^{2}=30 \mathrm{GeV}^{2}\end{array}$ \\
\hline $40-65$ & 52.5 & $5.63 \pm 0.58_{-0.35}^{+0.40}$ & $2.52 \pm 0.26_{-0.18}^{+0.09}$ & $0.99 \pm 0.13_{-0.10}^{+0.05}$ \\
$65-90$ & 77.5 & $6.57 \pm 0.91_{-0.81}^{+0.47}$ & $3.12 \pm 0.39_{-0.17}^{+0.21}$ & $1.34 \pm 0.17_{-0.09}^{+0.05}$ \\
$90-115$ & 102.5 & $9.5 \pm 1.5_{-1.4}^{+0.8}$ & $3.94 \pm 0.61_{-0.30}^{+0.32}$ & $1.91 \pm 0.30_{-0.12}^{+0.12}$ \\
$115-140$ & 127.5 & $7.6 \pm 1.6_{-0.6}^{+1.5}$ & $5.83 \pm 0.89_{-0.48}^{+0.49}$ & $1.64 \pm 0.47_{-0.15}^{+0.13}$ \\
\hline
\end{tabular}

Table 3: Values of the cross sections for the $\gamma^{*} p \rightarrow \gamma p$ DVCS process as a function of $W$ for the $e^{+} p$ data in three $Q^{2}$ ranges. Values are quoted at the centre of each $W$ bin and for the average $Q^{2}$ values obtained from GenDVCS. The first uncertainty is statistical and the second systematic. The systematic uncertainty due to the luminosity determination is not included. 
(a)

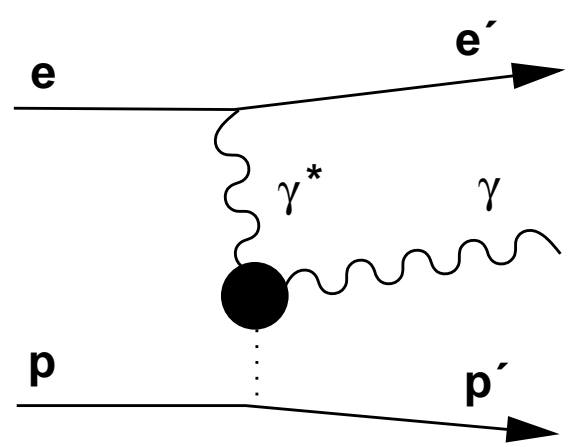

(b)

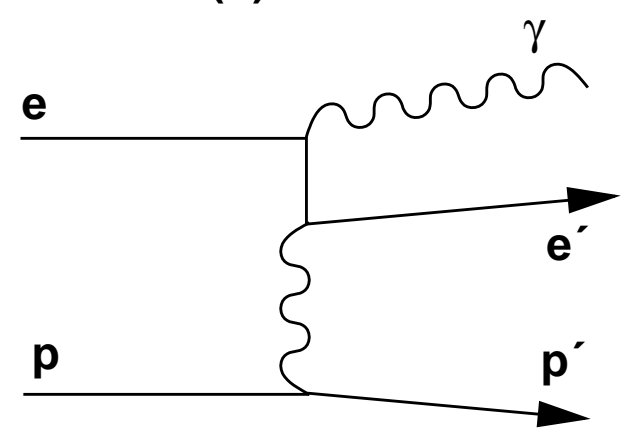

(c)

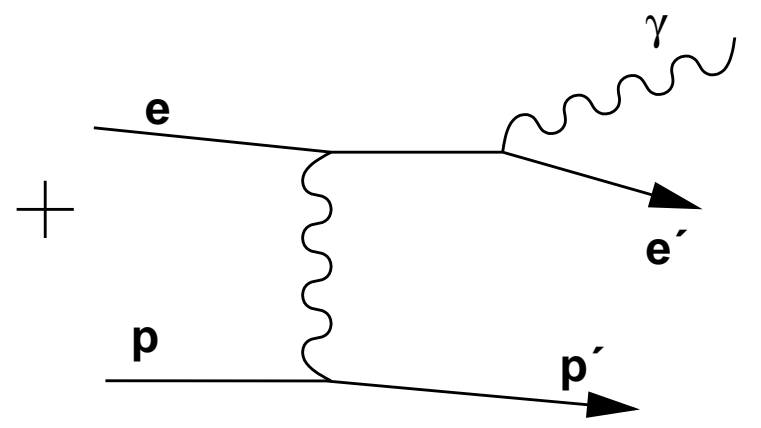

Figure 1: (a) Deeply virtual Compton scattering (DVCS) diagram, (b) and (c) Bethe-Heitler (BH) process. 


\section{ZEUS}

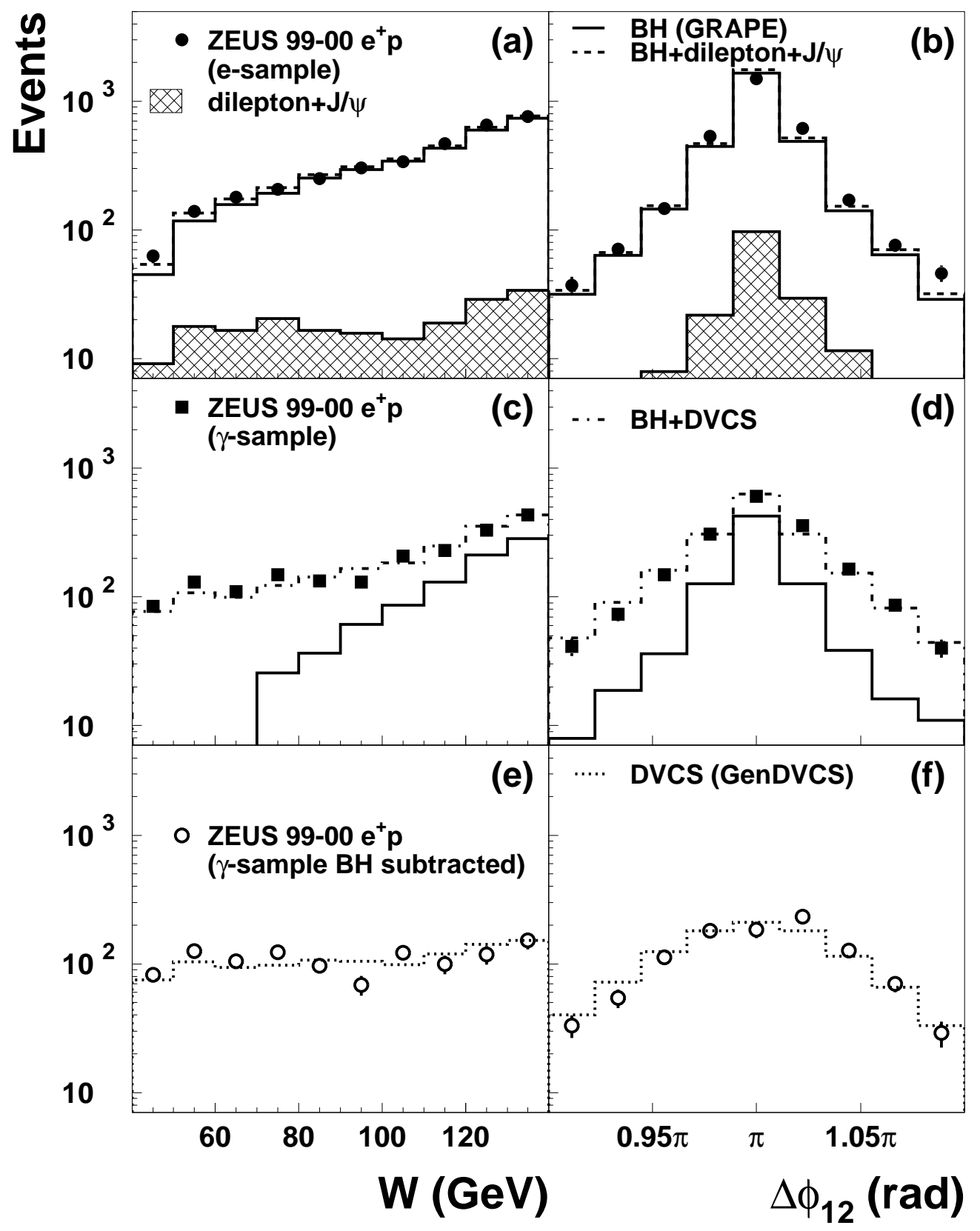

Figure 2: Comparison of the $W$ and the $\Delta \phi_{12}$ distributions for the data from 99-00 $e^{+} p$ running period and corresponding $M C$ samples as described in the figure; (a) and (b) e sample compared to the sum of the BH, dilepton and $J / \psi M C$ simulations, with the sum of the latter two also shown separately; (c) and (d) $\gamma$ sample compared to the expectation of the BH MC simulation, normalised to the data in the e sample after background subtraction; $(e)$ and $(f) \gamma$ sample after subtracting the BH expectation compared to GenDVCS, where the absolute normalisation of the latter is increased by $17.5 \%$ to account for the proton-dissociative component in the DVCS data. 


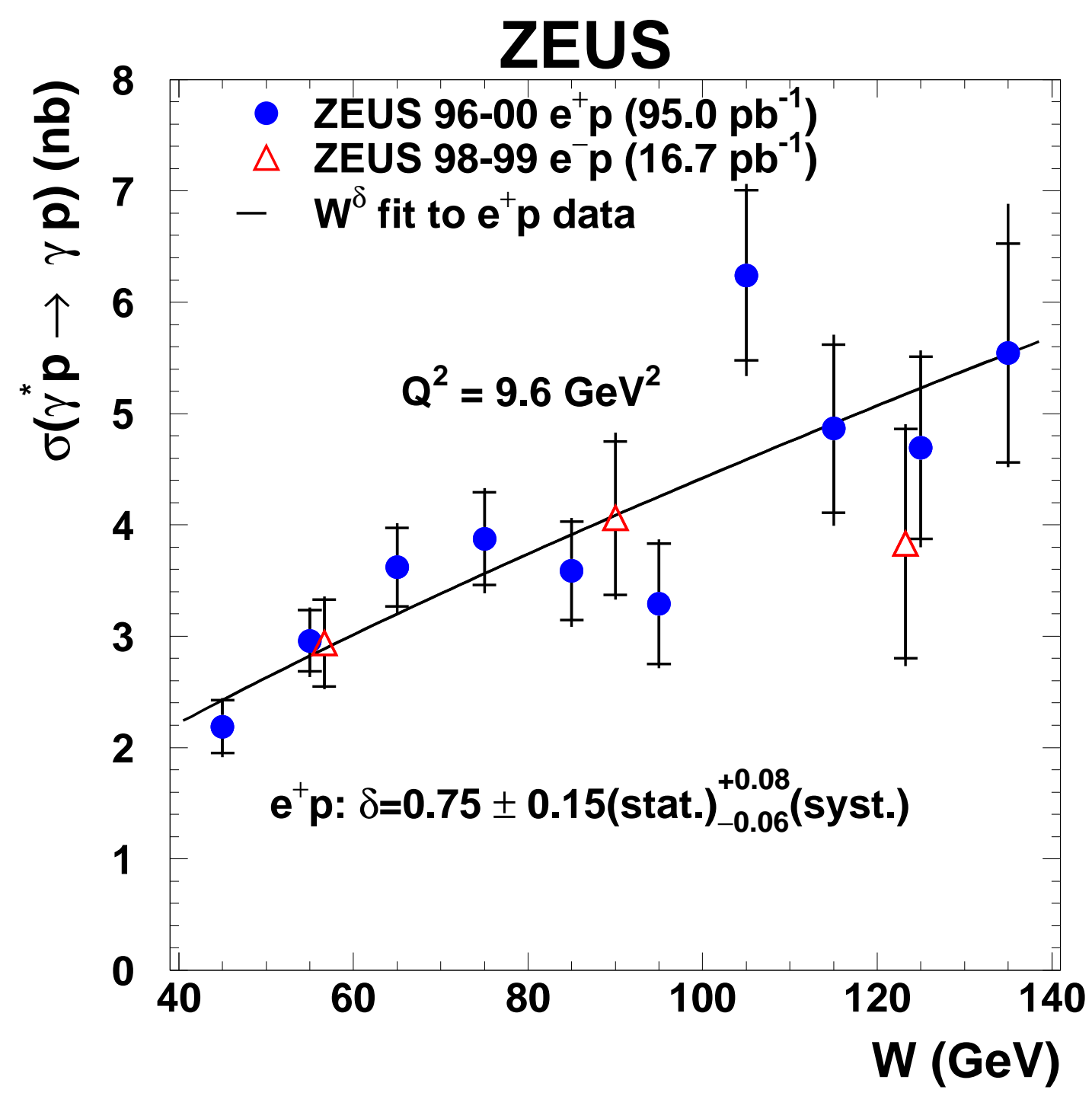

Figure 3: The DVCS cross section, $\sigma\left(\gamma^{*} p \rightarrow \gamma p\right)$, as a function of $W$ for an average $Q^{2}=9.6 \mathrm{GeV}^{2}$, separately for $e^{+} p$ data (dots) and $e^{-} p$ data (triangles). The solid line is the result of a fit of the form $\sigma_{\mathrm{DVCS}} \propto W^{\delta}$ to the positron data. The error bars denote the statistical uncertainty (inner) and the quadratic sum of the statistical and the systematic uncertainties (outer). 


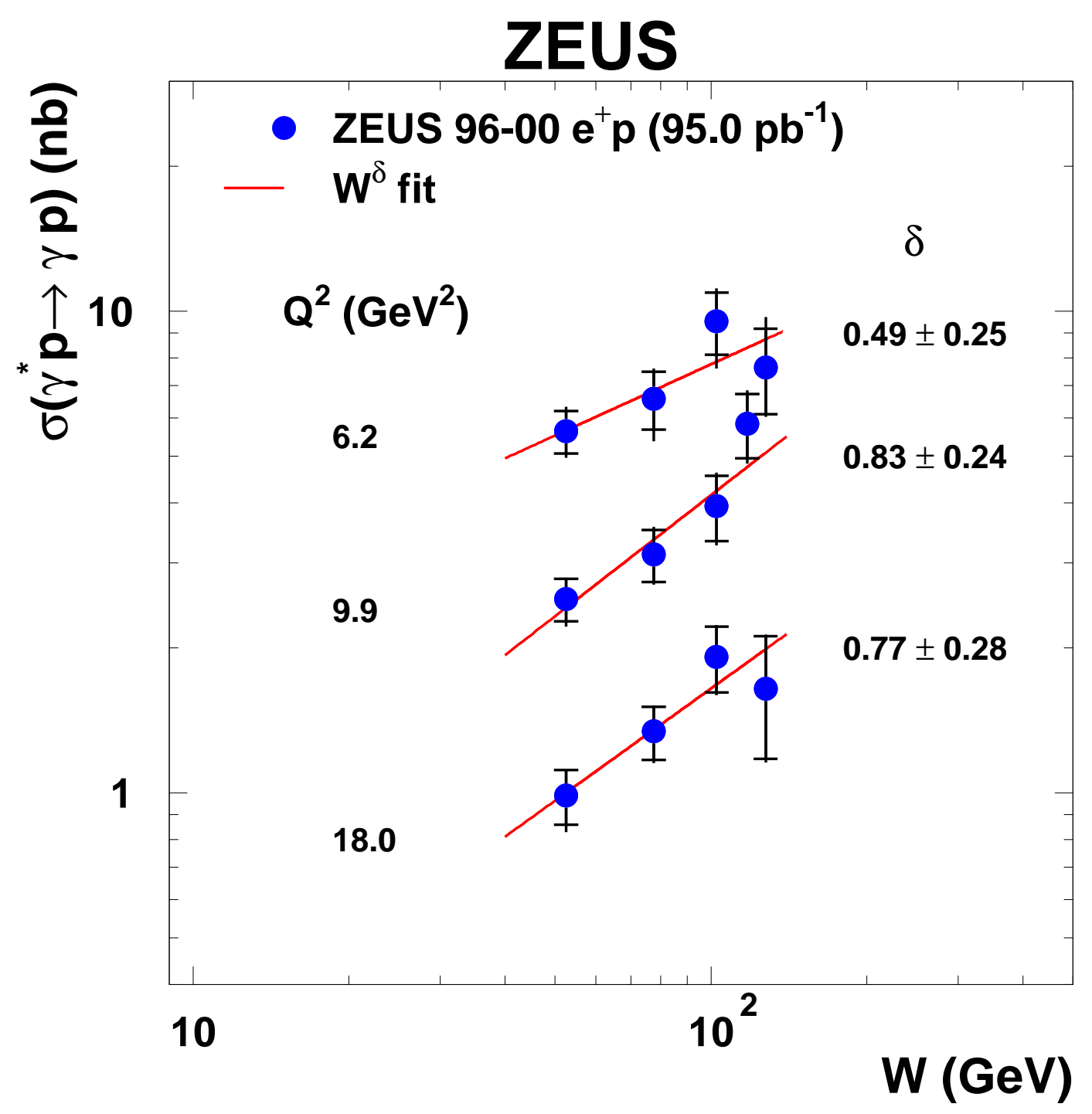

Figure 4: The DVCS cross section, $\sigma\left(\gamma^{*} p \rightarrow \gamma p\right)$, as a function of $W$ for three $Q^{2}$ values for $e^{+} p$ data as denoted in the figure. The corresponding ranges in $Q^{2}$ are listed in Table 3 . The solid line is the result of a fit of the form $\sigma_{\mathrm{DVCS}} \propto W^{\delta}$. The values of $\delta$ and their statistical uncertainties are given in the figure. The last data point for $Q^{2}=9.9 \mathrm{GeV}^{2}$ is displaced horizontally for ease of visibility. The error bars denote the statistical uncertainty (inner) and the quadratic sum of the statistical and the systematic uncertainties (outer). 


\section{ZEUS}

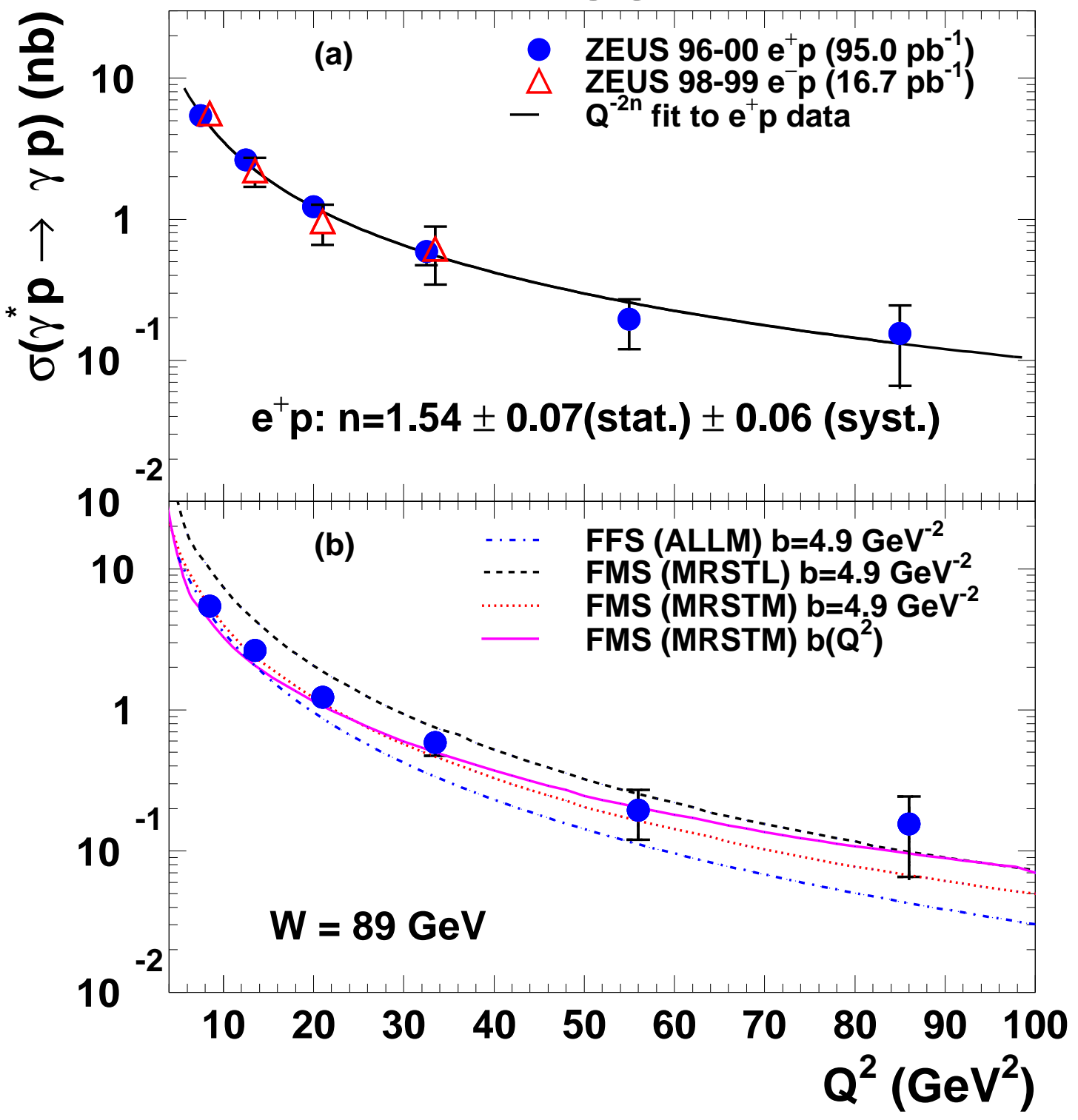

Figure 5: (a) The DVCS cross section, $\sigma\left(\gamma^{*} p \rightarrow \gamma p\right)$, as a function of $Q^{2}$ for $W=89 \mathrm{GeV}$, separately for $e^{+} p$ data (dots) and $e^{-} p$ data (triangles). The solid line is the result of a fit of the form $\sigma_{\mathrm{DVCS}} \propto Q^{-2 n}$ to the positron data. The $e^{-} p$ data points are displaced horizontally for ease of visibility; (b) $\sigma\left(\gamma^{*} p \rightarrow \gamma p\right)$ as a function of $Q^{2}$ compared to the GPD-based theoretical predictions of FFS and $F M S$, where MRSTL(M) indicates the LO (NLO) parameterisation of PDF. The MRSTM expectations are also shown for the $Q^{2}$-dependent $b$ values described in the text. The error bars denote the statistical uncertainty (inner) and the quadratic sum of the statistical and the systematic uncertainties (outer). 
ZEUS

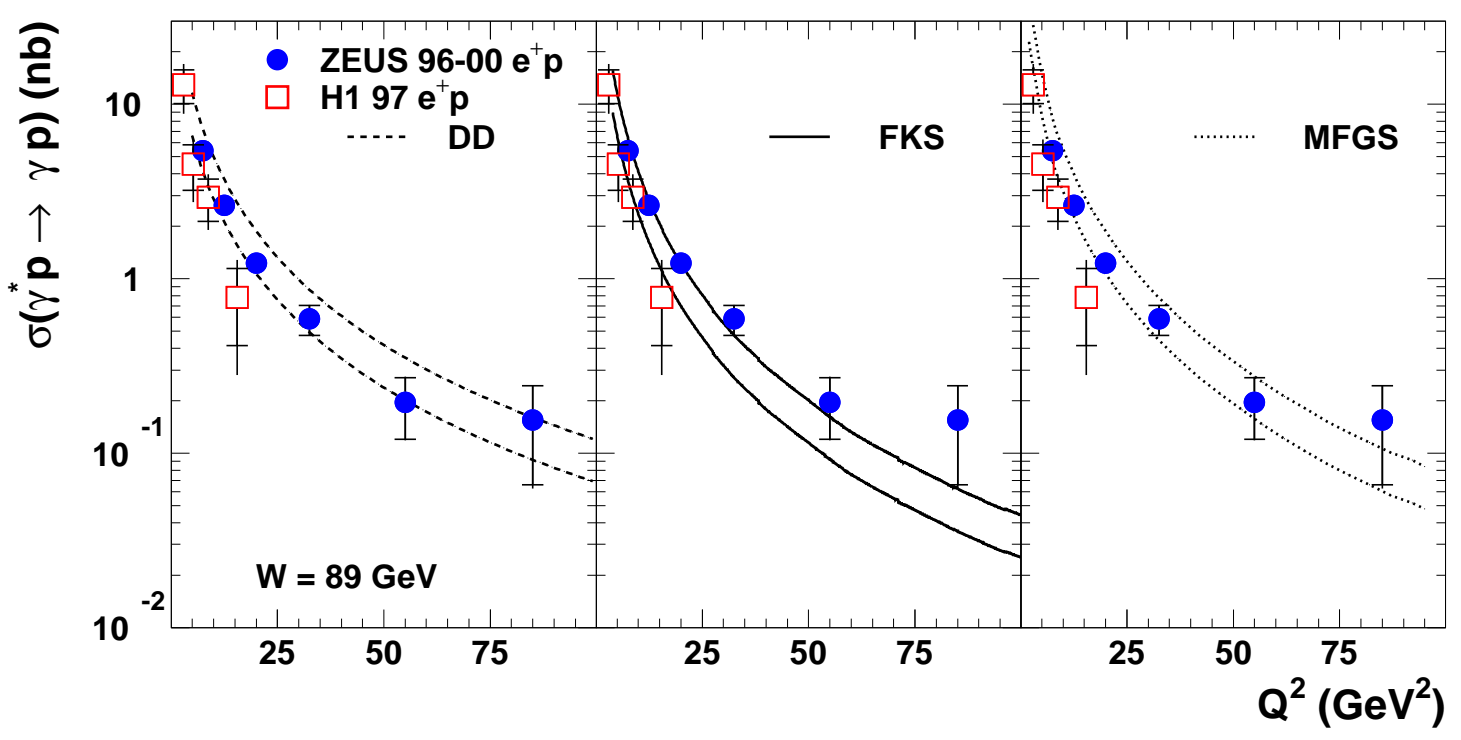

Figure 6: The DVCS cross section, $\sigma\left(\gamma^{*} p \rightarrow \gamma p\right)$, as a function of $Q^{2}$, as also shown in Fig. 國, for the ZEUS (dots) and H1 (squares) data. The data are compared to the theoretical predictions of the DD, FKS and MFGS models of colour-dipole interactions. The curves correspond to fixed $b$ values, $b=4 \mathrm{GeV}^{-2}$ (upper) and $b=7 \mathrm{GeV}^{-2}$ (lower). 